\title{
COMPOSITION OF MAJOR ORGANIC ACIDS IN VEGETABLES AND SPICES
}

\author{
Liga Priecina ${ }^{1}$, Daina Karklina ${ }^{2}$
}

\begin{abstract}
Organic acids are one of the major phytochemicals in vegetables and responsible for food taste and odor. Different organic acids are analyzed in fruits and cereals, but least in vegetables and spices. Organic acids has been analyzed because of their high importance in the formation of other phytochemical and increased antioxidant activity. The aim of the current research was to determine the oxalic, tartaric, quinic, malic, malonic, ascorbic, citric, fumaric, succinic, salicylic and benzoic acid content in fresh and pre-treated (with steam) vegetables and spices using high performance liquid chromatography (HPLC) method. Major organic acids in highest concentrations in spices and vegetables are quinic, malic, malonic and citric acids. Spices contain higher total organic acid content than vegetables. Using steaming as pre-treatment, some of the organic acids content significantly decreased. Obtained changes could be explained by the organic acid formation into more complex chemicals in food or metabolic process. For the future, these changes will be combined with individual phenolic compound changes in analyzed samples.
\end{abstract}

UDC Classification: 542.9, DOI: http://dx.doi.org/10.12955/cbup.v3.637

Keywords: Organic acids, vegetables, spices, steaming, HPLC

\section{Introduction}

Organic acids are typical products of cell metabolism. All organic acids occur naturally in a variety of vegetables and animal substrates, and can be present either as constituents of foods as a result of normal biochemical metabolic processes, direct addition as acidulates, hydrolysis or bacterial growth, or can be later added directly or indirectly in products (Theron \& Rykers, 2010). Organic acids are important to biological processes, since they are involved in various fundamental pathways in plant and animal metabolism and catabolism as intermediate or final products, playing a key role in the citric acid cycle or Krebs cycle (Gonzalez \& Gonzalez, 2012). The citric acid cycle (Krebs cycle or tricarboxylic acid cycle) is an important pathway in eukaryotes. It is the primary source of electrons (usually stemming from pyruvates) donating to the respiratory membrane in mitochondria (Schnarreberger \& Martin, 2002).

The incorporation of acids into food can shorten sterilization times for heat treatment, owing to the lowered heat resistance of microorganisms in foods with increased acidity. Toxicology, antimicrobial properties, application, and regulatory status are different for each food acid. The carboxylic acids are more polar than lipophilic acids and are traditionally used in foods or their secondary effects rather than for their ability to inhibit microbial growth. The presence of acid can effectively inhibit germination and outgrowth of spores that survive the thermal process. Salt, sugar, and occurring agents in conjunction with acids serve to further decrease processing times. These interactions ensure food sterility, but the processing time would also aid in preserving the palatability of the product (Doores, 2005).

Most raw vegetables are unpalatable and can undergo growing quality changes associated with enzymatic reactions; therefore, it is necessary to process vegetables to increase their shelf life and improve their eating quality. During processing, vegetables are often subjected to mechanical (peeling, cutting, mixing, homogenisation, coring, etc.) and thermal (blanching, etc.) treatments. These treatments and the sequences of performing them could influence the stability of vitamin $\mathrm{C}$ and other important nutrients during the treatments themselves and/or during subsequent processing, straight to occurrence of chemical and enzymatic oxidation reactions (Wambui Munyaka, Oey, Van Loey, \& Hendrickx, 2010).

\footnotetext{
${ }^{1}$ Liga Priecina, Faculty of Food Technology, Latvia University of Agriculture, Latvia, liga.priecina@gmail.com

${ }^{2}$ Daina Karklina, Faculty of Food Technology, Latvia University of Agriculture, Latvia, daina.karklina@1lu.lv
} 
The analysis of organic acids in different food products such as fruits, juices, vegetables, dairy products, coffee, and wine is of great interest for food industry since these compounds are responsible for sensory properties and may also influence their stability (Rodriguez-Bernaldo de Quiros, LageYusty, \& Lopez-Hernandez, 2009).

Monitoring organic acids simultaneously is often essential and is typically conducted with ion/liquid chromatography. Many samples contain fine particles, proteins, lipids, other small organic compounds, and macromolecules, which drastically affect the usable lifetime of chromatographic columns. Reported sample pre-treatment methods for liquid chromatography determination of organic acids in foods and beverages include filtration-centrifugation, solid phase extraction, and on-line dialysis (Ohira et al., 2014).

The aim of the current research was to determine the oxalic, tartaric, quinic, malic, malonic, ascorbic, citric, fumaric, succinic, salicylic, and benzoic acid content in fresh and pre-treated with steam vegetables and spices using high performance liquid chromatography (HPLC) method.

\section{Materials and methods}

\section{Reagents and Samples}

Reagents: oxalic, L-(+)-tartaric, quinic, L-(-) malic, malonic, L-(+)-ascorbic, citric, fumaric, succinic, salicylic, benzoic acid, acetonitrile, potassium dihydrogenphosphate $\left(\mathrm{KH}_{2} \mathrm{PO}_{4}\right)$, o-phosphoric acid, and m-phosphoric acid. All reagents are of the highest HPLC grade and analytical grade purity.

Samples: dill (Anethum graveolens), celery (Apium graveolens var. dulce), parsley (Petroselinum crispum), leek (Allium ampeloprasum L.), onion (Allium cepa), garlic (Allium sativum L.), celery root (Apium graveolens var. rapaceum), carrot (Daucus carota), and pumpkin (Cucurbita maxima) were harvested in Latvia, Zemgale region in 2014. Fresh samples were peeled and cut in equal-sized small pieces. Steaming was done using home steaming device, Tefal VC4003Vitamin+ (China), at the temperature of $95 \pm 3^{\circ} \mathrm{C}$ temperature for 1.5 and 3.0 minutes (Turkmen, Sari, \& Velioglu, 2005). After steaming, samples were taken out from the container and cooled down to the temperature of $20 \pm 2^{\circ} \mathrm{C}$. Fresh and steamed samples were blended with Braun Multiquick 5 (Germany).

\section{Moisture content}

The moisture content was determined according to the AOAC standard method (Mazzeo, N'Dri, Chiavaro, Visconti, \& Fogliano, 2011). Samples were dried at $105 \pm 1{ }^{\circ} \mathrm{C}$ for 2 hours. The moisture content was expressed as a percentage. Dry matter was calculated from moisture content.

\section{Sample preparation}

Approximately $10.00 \pm 0.01 \mathrm{~g}$ fresh and steamed samples were weighted in flask and $50.0 \mathrm{~mL}$ freshly prepared m-phosphoric acid in distilled water $(\mathrm{pH}=3.00 \pm 0.20)$ was pored over. Samples were mixed at $18 \pm 1^{\circ} \mathrm{C}$ temperature for 2 hours and then filtrated 2 times through Whatman filter paper and before injection in chromatograph with $0.45 \mu \mathrm{m}$ filter. A pH equal or lover than 5.00 is necessary in mobile phase to maintain the stability of vitamin $\mathrm{C}$ during the analysis (Martin-Belloso, Odrizola-Serrano, \& Soliva-Fortuny, 2012)

\section{High Performance Liquid Chromatography (HPLC) analysis}

Determination of oxalic, tartaric, quinic, malic, malonic, ascorbic, citric, fumaric, and succinic was carried out according to Scherer et al. (2012) with some modifications. We used Shimadzu LC-20 Prominence (Japan) liquid chromatograph, equipped with detector DAD SPD-M20A, auto sampler adjusted to $10 \mu \mathrm{L}$ volume injection, and Alltech C18 column $(5 \mu \mathrm{m}$ particle size, $250 \times 4.6 \mathrm{~mm}$, kept at $30^{\circ} \mathrm{C}$ temperature). The mobile phase consisted of acetonitrile and $0.05 \mathrm{M} \mathrm{KH}_{2} \mathrm{PO}_{4}$ (ratio 1:99) (pH 
$=2.80 \pm 0.02$ adjusted with o-phosphoric acid) using an isocratic elution with a flow rate $1.00 \mathrm{~mL}$ min

${ }^{-1}$. Detection was at $210 \mathrm{~nm}$. Total analysis time was up to 10 minutes.

Determination of salicylic and benzoic acid was carried out according to Bae et al. (2014) with some modifications. We used Shimadzu LC-20 Prominence (Japan) liquid chromatograph, equipped with detector DAD SPD-M20A, auto sampler adjusted to $10 \mu \mathrm{L}$ volume injection, and Alltech C18 column ( $5 \mu \mathrm{m}$ particle size, $250 \times 4.6 \mathrm{~mm}$, kept at $25^{\circ} \mathrm{C}$ temperature). The mobile phase consisted of acetonitrile and $0.025 \mathrm{M} \mathrm{KH}_{2} \mathrm{PO}_{4}$ (ratio 45:55) $(\mathrm{pH}=2.55 \pm 0.02$ adjusted with o-phosphoric acid) using a gradient elution with a flow rate $1.50 \mathrm{~mL} \mathrm{~min}^{-1}$. Detection was at $210 \mathrm{~nm}$. Total analysis time was up to 5.5 minutes.

\section{Statistical analysis}

The moisture content was carried out in triplicate and organic acid content in duplicate. Data were expressed as an average amount and standard deviation. Result analysis was prepared using Microsoft Excel 2010.

\section{Results and discussion}

All analyzed samples contain high amount of moisture. For further organic acid content, dry matter content was calculated (Table 1).

\begin{tabular}{|l|r|r|r|}
\hline \multicolumn{5}{|c|}{ Table 1: Dry matter content of fresh and steamed vegetables and spices (percent) } \\
\hline \multicolumn{1}{|c|}{ Sample } & \multicolumn{1}{c|}{ Fresh } & Steamed for 1.5 min & Steamed for 3.0 min \\
\hline Dill & $15.37 \pm 0.15$ & $15.36 \pm 0.15$ & $14.31 \pm 0.14$ \\
\hline Celery & $19.18 \pm 0.19$ & $18.58 \pm 0.19$ & $18.60 \pm 0.19$ \\
\hline Parsley & $21.02 \pm 0.21$ & $19.85 \pm 0.20$ & $20.82 \pm 0.21$ \\
\hline Leek & $11.80 \pm 0.12$ & $10.29 \pm 0.10$ & $12.10 \pm 0.12$ \\
\hline Onion & $6.18 \pm 0.06$ & $5.69 \pm 0.06$ & $6.01 \pm 0.06$ \\
\hline Garlic & $35.98 \pm 0.36$ & $34.80 \pm 0.35$ & $35.66 \pm 0.36$ \\
\hline Celery root & $11.05 \pm 0.11$ & $11.10 \pm 0.11$ & $11.31 \pm 0.11$ \\
\hline Carrot & $10.22 \pm 0.10$ & $10.68 \pm 0.11$ & $11.37 \pm 0.11$ \\
\hline Pumpkin & $5.63 \pm 0.06$ & $5.75 \pm 0.06$ & $6.03 \pm 0.06$ \\
\hline Source: Aun & & &
\end{tabular}

Source: Authors

There is no significant increase in dry matter content for the analyzed samples because steaming in the chamber does not add extra moisture to them.

Using steaming as pre-treatment, organic acid content decreases compared with fresh samples; in rare cases, content increases (Table 2)

\begin{tabular}{|c|c|c|c|c|c|c|c|c|c|c|c|c|}
\hline Samples & & 1. & 2. & 3. & 4. & 5. & 6. & 7. & 8. & 9. & 10. & 11. \\
\hline \multirow[t]{3}{*}{ Dill } & A & 349.71 & n.d. & $\begin{array}{l}7398 . \\
18\end{array}$ & $\begin{array}{l}5312 . \\
60\end{array}$ & n.d. & $\begin{array}{l}220.2 \\
9\end{array}$ & $\begin{array}{l}1941 . \\
73\end{array}$ & $\begin{array}{l}273 . \\
23\end{array}$ & 32.37 & 6.11 & $\begin{array}{l}1.8 \\
6\end{array}$ \\
\hline & B & 245.55 & $\begin{array}{l}674.8 \\
9\end{array}$ & $\begin{array}{l}960.9 \\
3\end{array}$ & n.d. & $\begin{array}{l}2338 . \\
74\end{array}$ & $\begin{array}{l}458.1 \\
4\end{array}$ & $\begin{array}{l}736.9 \\
2\end{array}$ & n.d. & 21.25 & 7.38 & $\begin{array}{l}7.5 \\
4\end{array}$ \\
\hline & $\mathrm{C}$ & 280.36 & $\begin{array}{l}141.0 \\
2\end{array}$ & n.d. & n.d. & $\begin{array}{l}3167 . \\
41\end{array}$ & $\begin{array}{l}925.6 \\
8\end{array}$ & $\begin{array}{l}775.1 \\
3\end{array}$ & n.d. & 25.72 & $\begin{array}{l}12.1 \\
1\end{array}$ & $\begin{array}{l}2.8 \\
9\end{array}$ \\
\hline
\end{tabular}


CBU INTERNATIONAL CONFERENCE ON INNOVATION, TECHNOLOGY TRANSFER AND EDUCATION

\begin{tabular}{|c|c|c|c|c|c|c|c|c|c|c|c|c|}
\hline \multirow[t]{3}{*}{ Celery } & A & 244.37 & n.d. & $\begin{array}{l}714.2 \\
4\end{array}$ & n.d. & $\begin{array}{l}4924 . \\
47\end{array}$ & $\begin{array}{l}221.2 \\
4\end{array}$ & $\begin{array}{l}4011 . \\
61\end{array}$ & n.d. & $\begin{array}{l}267.6 \\
0\end{array}$ & $\begin{array}{l}21.6 \\
2\end{array}$ & $\begin{array}{l}4.7 \\
3\end{array}$ \\
\hline & B & 152.00 & n.d. & $\begin{array}{l}781.7 \\
0\end{array}$ & $\begin{array}{l}3930 . \\
85\end{array}$ & n.d. & $\begin{array}{l}125.8 \\
8\end{array}$ & $\begin{array}{l}3632 . \\
83\end{array}$ & n.d. & 31.23 & $\begin{array}{l}34.3 \\
2\end{array}$ & $\begin{array}{l}3.1 \\
6\end{array}$ \\
\hline & $\mathrm{C}$ & 152.91 & $\begin{array}{l}231.6 \\
2\end{array}$ & $\begin{array}{l}775.7 \\
4\end{array}$ & $\begin{array}{l}1679 . \\
30\end{array}$ & n.d. & 88.89 & $\begin{array}{l}958.4 \\
6\end{array}$ & n.d. & 17.92 & $\begin{array}{l}16.6 \\
4\end{array}$ & $\begin{array}{l}1.7 \\
8\end{array}$ \\
\hline \multirow[t]{3}{*}{ Parsley } & A & 479.64 & $\begin{array}{l}733.5 \\
5\end{array}$ & $\begin{array}{l}1168 . \\
87\end{array}$ & n.d. & $\begin{array}{l}1748 . \\
02\end{array}$ & 85.66 & $\begin{array}{l}1940 . \\
34\end{array}$ & n.d. & $\begin{array}{l}273.0 \\
5\end{array}$ & $\begin{array}{l}72.8 \\
5\end{array}$ & $\begin{array}{l}0.2 \\
1\end{array}$ \\
\hline & B & 182.07 & $\begin{array}{l}293.3 \\
4\end{array}$ & $\begin{array}{l}738.4 \\
6\end{array}$ & 79.88 & 58.93 & $\begin{array}{l}647.5 \\
7\end{array}$ & $\begin{array}{l}407.4 \\
3\end{array}$ & n.d. & 16.04 & $\begin{array}{l}29.2 \\
6\end{array}$ & $\begin{array}{l}2.3 \\
7\end{array}$ \\
\hline & $\mathrm{C}$ & 203.30 & $\begin{array}{l}775.4 \\
5\end{array}$ & $\begin{array}{l}179.6 \\
9\end{array}$ & n.d. & $\begin{array}{l}126.8 \\
9\end{array}$ & $\begin{array}{l}575.1 \\
8\end{array}$ & $\begin{array}{l}850.7 \\
1\end{array}$ & n.d. & 51.32 & $\begin{array}{l}37.4 \\
0\end{array}$ & $\begin{array}{l}2.3 \\
8\end{array}$ \\
\hline \multirow[t]{3}{*}{ Leek } & A & 101.85 & n.d. & 94.44 & n.d. & 60.10 & 32.68 & $\begin{array}{l}1532 . \\
55\end{array}$ & 0.46 & 36.41 & n.d. & $\begin{array}{l}0.7 \\
9\end{array}$ \\
\hline & B & 75.92 & $\begin{array}{l}127.6 \\
3\end{array}$ & $\begin{array}{l}1889 . \\
45\end{array}$ & $\begin{array}{l}935.4 \\
4\end{array}$ & n.d. & 43.74 & $\begin{array}{l}363.8 \\
3\end{array}$ & $\begin{array}{l}23.8 \\
3\end{array}$ & 45.98 & 0.42 & $\begin{array}{l}0.2 \\
3\end{array}$ \\
\hline & $\mathrm{C}$ & 73.17 & $\begin{array}{l}995.9 \\
7\end{array}$ & n.d. & $\begin{array}{l}1391 . \\
46\end{array}$ & n.d. & $\begin{array}{l}154.0 \\
2\end{array}$ & $\begin{array}{l}648.0 \\
2\end{array}$ & 0.45 & 34.29 & 0.53 & $\begin{array}{l}n . \\
d .\end{array}$ \\
\hline \multirow[t]{3}{*}{ Carrot } & A & 316.46 & $\begin{array}{l}840.6 \\
3\end{array}$ & n.d. & $\begin{array}{l}168.1 \\
9\end{array}$ & n.d. & 27.84 & $\begin{array}{l}1074 . \\
49\end{array}$ & $\begin{array}{l}104 . \\
31\end{array}$ & 14.57 & n.d. & $\begin{array}{l}1.8 \\
3\end{array}$ \\
\hline & B & 228.07 & $\begin{array}{l}776.1 \\
0\end{array}$ & n.d. & $\begin{array}{l}301.4 \\
2\end{array}$ & n.d. & 23.71 & $\begin{array}{l}321.9 \\
4\end{array}$ & 9.98 & 79.63 & 0.60 & $\begin{array}{l}0.4 \\
2\end{array}$ \\
\hline & $\mathrm{C}$ & 139.49 & $\begin{array}{l}559.8 \\
2\end{array}$ & $\begin{array}{l}1030 . \\
42\end{array}$ & $\begin{array}{l}1818 . \\
20\end{array}$ & n.d. & 34.11 & $\begin{array}{l}215.7 \\
9\end{array}$ & $\begin{array}{l}22.0 \\
7\end{array}$ & n.d. & n.d. & $\begin{array}{l}0.7 \\
8\end{array}$ \\
\hline \multirow[t]{3}{*}{$\begin{array}{l}\text { Pumpki } \\
\text { n }\end{array}$} & A & $\begin{array}{l}15011 . \\
17\end{array}$ & n.d. & n.d. & $\begin{array}{l}2491 . \\
77\end{array}$ & n.d. & 8.31 & $\begin{array}{l}1914 . \\
29\end{array}$ & $\begin{array}{l}294 . \\
17\end{array}$ & 12.38 & 1.19 & $\begin{array}{l}0.2 \\
5\end{array}$ \\
\hline & B & $\begin{array}{l}8026.6 \\
3\end{array}$ & n.d. & $\begin{array}{l}1502 . \\
85\end{array}$ & n.d. & $\begin{array}{l}1513 . \\
91\end{array}$ & $\begin{array}{l}933.2 \\
9\end{array}$ & $\begin{array}{l}688.4 \\
2\end{array}$ & 3.57 & $\begin{array}{l}1135 . \\
03\end{array}$ & 0.94 & $\begin{array}{l}0.4 \\
3\end{array}$ \\
\hline & $\mathrm{C}$ & 554.13 & $\begin{array}{l}1542 . \\
56\end{array}$ & $\begin{array}{l}8313 . \\
43\end{array}$ & n.d. & $\begin{array}{l}2799 . \\
45\end{array}$ & $\begin{array}{l}1827 . \\
59\end{array}$ & $\begin{array}{l}891.7 \\
6\end{array}$ & 6.84 & 3.94 & 2.33 & $\begin{array}{l}0.8 \\
8\end{array}$ \\
\hline \multirow[t]{3}{*}{$\begin{array}{l}\text { Celery } \\
\text { root }\end{array}$} & A & 806.75 & n.d. & n.d. & n.d. & $\begin{array}{l}3363 . \\
54\end{array}$ & $\begin{array}{l}243.0 \\
8\end{array}$ & $\begin{array}{l}5465 . \\
71\end{array}$ & 0.34 & $\begin{array}{l}7597 . \\
84\end{array}$ & n.d. & $\begin{array}{l}3.9 \\
7\end{array}$ \\
\hline & $\mathrm{B}$ & 173.12 & n.d. & $\begin{array}{l}795.9 \\
2\end{array}$ & n.d. & $\begin{array}{l}169.1 \\
2\end{array}$ & $\begin{array}{l}1516 . \\
56\end{array}$ & $\begin{array}{l}1658 \\
36\end{array}$ & 3.61 & 8.00 & 1.08 & $\begin{array}{l}8.4 \\
8\end{array}$ \\
\hline & $\mathrm{C}$ & 737.26 & n.d. & n.d. & n.d. & $\begin{array}{l}2890 . \\
69\end{array}$ & $\begin{array}{l}1002 . \\
08\end{array}$ & $\begin{array}{l}1431 . \\
20\end{array}$ & 1.01 & $\begin{array}{l}1262 . \\
42\end{array}$ & 1.05 & $\begin{array}{l}1.7 \\
5\end{array}$ \\
\hline \multirow[t]{3}{*}{ Onion } & A & 474.66 & $\begin{array}{l}1542 . \\
64\end{array}$ & n.d. & n.d. & $\begin{array}{l}1247 . \\
42\end{array}$ & 10.32 & $\begin{array}{l}281.9 \\
7\end{array}$ & $\begin{array}{l}34.7 \\
3\end{array}$ & $\begin{array}{l}441.5 \\
9\end{array}$ & $\begin{array}{l}24.6 \\
3\end{array}$ & $\begin{array}{l}0.2 \\
3\end{array}$ \\
\hline & B & 370.66 & $\begin{array}{l}1178 . \\
39\end{array}$ & n.d. & n.d. & 22.93 & 23.59 & $\begin{array}{l}461.8 \\
9\end{array}$ & 1.23 & 22.11 & $\begin{array}{l}25.8 \\
8\end{array}$ & $\begin{array}{l}n . \\
d .\end{array}$ \\
\hline & $\mathrm{C}$ & 547.61 & $\begin{array}{l}2256 . \\
15\end{array}$ & n.d. & n.d. & n.d. & 25.25 & $\begin{array}{l}664.9 \\
2\end{array}$ & 1.03 & n.d. & $\begin{array}{l}15.1 \\
2\end{array}$ & $\begin{array}{l}0.3 \\
2\end{array}$ \\
\hline \multirow[t]{3}{*}{ Garlic } & A & 803.59 & $\begin{array}{l}608.0 \\
4\end{array}$ & $\begin{array}{l}1565 . \\
95\end{array}$ & n.d. & $\begin{array}{l}124.7 \\
3\end{array}$ & 15.57 & $\begin{array}{l}215.0 \\
4\end{array}$ & 4.82 & $\begin{array}{l}513.5 \\
4\end{array}$ & $\begin{array}{l}14.0 \\
8\end{array}$ & $\begin{array}{l}n . \\
d .\end{array}$ \\
\hline & $\mathrm{B}$ & 892.66 & $\begin{array}{l}851.9 \\
3\end{array}$ & $\begin{array}{l}1204 . \\
65\end{array}$ & n.d. & $\begin{array}{l}319.0 \\
0\end{array}$ & $\begin{array}{l}2263 . \\
49\end{array}$ & $\begin{array}{l}340.8 \\
8\end{array}$ & 0.11 & $\begin{array}{l}266.7 \\
7\end{array}$ & 0.51 & $\begin{array}{l}n . \\
d .\end{array}$ \\
\hline & $\mathrm{C}$ & 829.60 & $\begin{array}{l}992.4 \\
7\end{array}$ & $\begin{array}{l}1376 . \\
40\end{array}$ & n.d. & $\begin{array}{l}572.2 \\
4\end{array}$ & $\begin{array}{l}2623 . \\
13\end{array}$ & $\begin{array}{l}418.8 \\
1\end{array}$ & 2.12 & $\begin{array}{l}102.4 \\
3\end{array}$ & n.d. & n.d \\
\hline
\end{tabular}

Note: A- fresh samples; B- steamed $1.5 \mathrm{~min}$; C- steamed $3.0 \mathrm{~min}$. 1.-Oxalic acid; 2.-Tartaric acid; 3.- Quinic acid; 4.- Malic acid; 5.-Malonic acid; 6.-Ascorbic acid; 7.-Citric acid; 8.- Fumaric acid; 
9.- Succinic acid; 10.-Salicylic acid; 11.-Benzoic acid. n.d.- not detected. Results, average amount and standard deviation (10\%), was expressed as milligrams per $100 \mathrm{~g}$ dry weight (mg $100 \mathrm{~g} \mathrm{~g}^{-1} \mathrm{DW}$ ).

Source: Authors

In dills, the main organic acids are quinic, malic, and citric acid, and their content significant decreases after steaming. Malonic and benzoic content increases using steaming as pre-treatment. Main organic acids in celeries are malonic (after steaming, acid was not detected) and citric acid (content decreases after steaming). Malic acid was not detected in fresh samples, but significant amount was detected in steamed sample after $1.5 \mathrm{~min}$, and then after $3.0 \mathrm{~min}$ steamed content decreases.

In parsleys, the highest organic acid contents determined were quinic, malonic, and citric acids; their content decreases significantly after steaming for $1.5 \mathrm{~min}$, but malonic and citric acid content increases after 3.0 min treatment with steam. Malic acid was detected only after $1.5 \mathrm{~min}$ of steaming; in other sample preparations it was not. Ascorbic acid content increases after steaming for $1.5 \mathrm{~min}$, then decreases after $3.0 \mathrm{~min}$.

In leeks, the main acids are quinic and citric acid; the highest quinic acid content was determined after being steamed for $1.5 \mathrm{~min}$, and was not detected after $3.0 \mathrm{~min}$. Tartaric, malic, and ascorbic acid content significant increases after steaming for $3.0 \mathrm{~min}$. Leek contains high concentrations of quinic and malic acid (after steaming for $1.5 \mathrm{~min}$, concentration increases for both, but decreases for quinic acid after $3.0 \mathrm{~min}$ ). Citric acid decreases after steaming. Malonic acid concentration was relatively lower and was not detected after steaming.

In carrots, the major organic acids were malic acid (concentration increases after steaming) and citric acid (concentration decreases after steaming). Malonic acid was not detected in carrots.

In pumpkins, the major organic acids were oxalic acid and citric acid (after steaming, concentration decreases), quinic acid, ascorbic acid (concentration increases after steaming), and succinic acid (concentration increases after $1.5 \mathrm{~min}$ of steaming, then decreases after $3.0 \mathrm{~min}$ ). Malonic acid was not detected in fresh samples, but concentration significantly increased after steaming. Malic acid was not detected.

In celery roots, malonic acid and succinic acid were detected in high concentrations. Concentration decreased after $1.5 \mathrm{~min}$ of steaming, but increased after $3.0 \mathrm{~min}$ of steaming. Citric acid concentration decreased after steaming. Tartaric and malic acid were not detected in celery roots.

In onions, malonic and succinic acid were in high concentrations. Content decreased after steaming for $1.5 \mathrm{~min}$, and were not detected after $3.0 \mathrm{~min}$. Citric acid concentration significantly increased after steaming for $3.0 \mathrm{~min}$. Tartaric acid concentration decreased after $1.5 \mathrm{~min}$ but increased after $3.0 \mathrm{~min}$. Quinic and malic acid were not detected in fresh and steamed onions.

In garlics, ascorbic acid content increases after steaming. Quinic and succinic acid content decreased after steaming for $1.5 \mathrm{~min}$, but quinic acid content increased after $3.0 \mathrm{~min}$ of steaming. Oxalic acid content decreased after 3.0 min of steaming. Malic and benzoic acid were not detected in fresh and steamed garlics.

According to Gamboa-Santos et al. (2013), vitamin C (ascorbic acid plus dehydroascorbic acid)

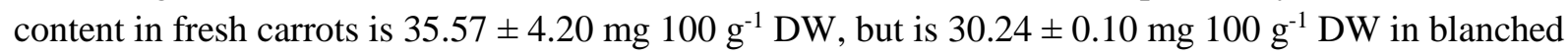
carrots. In case of blanching carrots at $95^{\circ} \mathrm{C}$ for 90 seconds, vitamin $\mathrm{C}$ retention was at $87.6 \%$. All of the results obtained from this research show that there are great influences of blanching conditions (temperature, time, sample geometry, blanching water, carrot weight ratio, etc.) on preservation of vitamin C content in carrots. According to Soininen et al. (2014), onion contains $5.51 \pm 3.94 \mathrm{~g} \mathrm{~kg}$ 
malic acid and $0.15 \pm 0.23 \mathrm{~g} \mathrm{~kg}$ fumaric acid; garlic contains $15.05 \pm 4.22 \mathrm{~g}$ kg malic acid and $0.02 \pm$ $0.02 \mathrm{~g}$ kg fumaric acid; leek contains $2.38 \pm 0.97 \mathrm{~g} \mathrm{~kg}$ malic acid and $0.77 \pm 0.37 \mathrm{~g} \mathrm{~kg}$ fumaric acid.

According to Mukherjee \& Chattopadhyay (2007), the ascorbic acid loss during hot water blanching is described as a diffusion controlled phenomenon. Increasing temperature in potato tissue from 50 to $100^{\circ} \mathrm{C}$ causes ascorbic acid diffusion to increase significantly.

According to Angumeenal \& Venkappayya (2013), increased temperature over $30^{\circ} \mathrm{C}$ has been found to increase oxalic acid content accumulation irrespective of the fermentation conditions. Therefore, maintenance of favored temperature is important for citric acid biosynthesis.

According to Kumar (2014), salicylic acid in plants is synthesized in plastids via two routes from chorismate, a product of shikimate pathway. One route is through isochorismate synthase, which is believed to be responsible for more than $90 \%$ of salicylic acid synthesized during activation of stress response. The other route is the phenylalanine ammonia-lyase mediated pathway. Salicylic acid is readily modified to its many derivatives (via glycosylation, methylations, amino acid conjugation, sulphonation, hydroxylation, etc.), but most of them are not active compounds.

According to Lindquist \& Yang (2011), benzoic acid remained stable even at $300^{\circ} \mathrm{C}$. Further benzoic acid stability experiments at higher temperature revealed that only $4 \%$ benzoic acid was degraded at $350^{\circ} \mathrm{C}$ with heat time for 10 minutes.

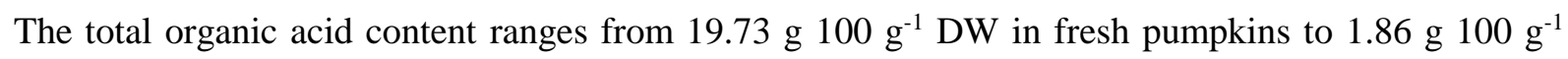
DW in fresh leeks.

\begin{tabular}{|l|r|r|r|}
\hline \multicolumn{4}{|c|}{ Table 3: Total organic acid content of fresh and steamed vegetables and spices (g $\left.100 \mathrm{~g}^{-1} \mathrm{DW}\right)$} \\
\hline \multicolumn{1}{|c|}{ Sample } & Fresh & Steamed for 1.5 min & Steamed for 3.0 min \\
\hline Dill & $15.54 \pm 1.55$ & $5.45 \pm 0.55$ & $5.33 \pm 0.53$ \\
\hline Celery & $10.41 \pm 1.04$ & $8.69 \pm 0.87$ & $3.92 \pm 0.39$ \\
\hline Parsley & $6.50 \pm 0.65$ & $2.46 \pm 0.25$ & $2.80 \pm 0.28$ \\
\hline Leek & $1.86 \pm 0.19$ & $3.51 \pm 0.35$ & $3.30 \pm 0.33$ \\
\hline Carrot & $2.55 \pm 0.26$ & $1.74 \pm 0.17$ & $3.82 \pm 0.38$ \\
\hline Pumpkin & $19.73 \pm 1.97$ & $13.81 \pm 1.38$ & $15.94 \pm 1.59$ \\
\hline Celery root & $17.48 \pm 1.75$ & $4.33 \pm 0.43$ & $7.33 \pm 0.73$ \\
\hline Onion & $4.06 \pm 0.41$ & $2.11 \pm 0.21$ & $3.51 \pm 0.35$ \\
\hline Garlic & $3.87 \pm 0.39$ & $6.14 \pm 0.61$ & $6.92 \pm 0.69$ \\
\hline Sourc: Aun & & & \\
\hline
\end{tabular}

Source: Authors

In some of the analyzed samples, steaming decreases the total organic acid content. In dill, content decreases by $64.9 \%$ and $65.7 \%$ after 1.5 and 3.0 minutes of steam treatment, respectively. Content in celery decreases by $16.5 \%$ and $62.3 \%$. Content in parsley decreases by $62.2 \%$ and $56.9 \%$ compared with fresh samples. In leeks, total organic acid content increases by $47.0 \%$ and $43.6 \%$ using steaming process compared to fresh ones. In carrots, the total content decreases by $31.8 \%$ after 1.5 minute of steaming but increases by $33.2 \%$ after 3.0 minutes of steaming compared to fresh ones. In pumpkins, the total content decreases by $30.0 \%$ and $19.2 \%$ using steaming process for 1.5 and 3.0 minutes, respectively, compared to fresh ones. In celery roots, content decreases by $75.2 \%$ and $58.1 \%$ using steaming compared to fresh ones. In onions, content decreases by $48.0 \%$ and $13.5 \%$ using steam compared to fresh ones. In garlic, content increases by $37.0 \%$ and $44.1 \%$ using steam treatment 
compared to fresh ones. These changes could be explained by organic acid degradation to form more complex compounds as well as other processes, e.g. oxidation.

According to Lopez-Bucio, Nieto-Jacobo, Ramirez-Rodriguez, \& Herrera-Estrella (2000), the total content of organic acids in plants is higher than in other organisms. The composition of organic acids that accumulates varies depending upon the species, age of plant, and the tissue type. The high accumulation of organic acids in plant tissues is most probably due to their important role as photosynthetic intermediates. Levels of organic acids vary between species, cultivars, or even among individual tissues of a plant when grown under identical conditions. Moreover, the biosynthesis, accumulation, transport, and root exudation of organic acids are dramatically increased in response to environmental stress.

\section{Conclusion}

Major organic acids in highest concentrations in spices and vegetables are quinic, malic, malonic, and citric acids. Using steaming as pre-treatment, some of the organic acids content significantly decreased. The highest ascorbic acid sources are steamed garlic, pumkins, and celery roots, while the best source of quinic acid are all analyzed samples, but not onions. Acorbic acid is important as natural antioxidant in vegetables and spices, while quinic acid is important compound for formation shikimic acid derivatives (phenolic compounds) in plants. Fresh dills, pumpkins, celeries and celery roots contain higher total organic acid content than other analyzed samples. Total organic acid content decreases significantly after using steam in most of analyzed samples with some exceptions. Obtained changes could be explained by organic acid formation into more complex chemicals in food or metabolic process. All analyzed vegetables and spices could be considered as excellent organic acid and antioxidant sources in nutrition. In the future, these changes will be combined with individual phenolic compound changes in analyzed samples. Most important analyzed organic acids in phenolic compound biosynthesis branch are quinic acid, salicylic, and benzoic acids to explain possible thermal treatment effect on phenolic compound formation and changes.

\section{Acknowledgment}

The research was realized with "Agricultural Resources for Sustainable Production of Qualitative and Healthy Foods in Latvia (AgroBioRes) (2014-2017)"; project No. 4 Sustainable use of local agricultural resources for qualitative and healthy food product development (FOOD) support.

\section{References}

Angumeenal, A.R., \& Venkappayya, D. (2013). An overview of citric acid production. LWT- Food Science and Technology, 50, 367-370.

Bae, H., Kyon Yun, S., Hae Jun, J., Koo Yoon, I., Young Nam, E., \& Hyun Kwon, J. (2014). Assesment of organic acid and sugar composition in apricot, plumcot, plum, and peach during fruit development. Journal of Applied Botany and Food Quality, 87, 24-29.

Doores, S., Davidson, P. M., Sofos, J. N., \& Branen, A. L. (2005). Organic acids. In: CRC Press Taylor \& Francis Group (Eds.), Antimicrobials in Food (3rd ed.) (pp. 91-142).

Gamboa-Santos, J., Soria, A. C., Perez-Mateos, M., Carrasco, J. A., Montilla, A., \& Villamiel, M. (2013). Vitamin C content and sensorial properties of dehydrated carrots blanched conventionally or by ultrasound. Food Chemistry, 136, 782-788.

Gonzalez, M., \& Gonzalez V. (2012). Organic acids. In: L. M. L. Nollet, F. Toldra, \& CRC Press Taylor \& Francis Group (Eds.), Food Analysis by HPLC (3rd ed.) (pp. 443-466).

Kumar, D. (2014). Salicylic acid signaling in disease resistance. Plant Science, 228, 127-134.

Lindquist, E., \& Yang, Y. (2011). Degradation of benzoic acid and its derivatives in subcritical water. Journal of Chromatography A, 1218, 2146-2152. 
Lopez-Bucio, J., Nieto-Jacobo, M. F., Ramirez-Rodriguez, V., \& Herrera-Estrella, L. (2000). Organic acid metabolism in plants: from adaptive physiology to transgenic varieties from cultivation in extreme soils. Plant Science, 160, 1-13.

Martin-Belloso, O., Odrizola-Serrano, I., \& Soliva-Fortuny, R. (2012). Vitamin C. In: L. M. L. Nollet, F. Toldra, \& CRC Press Taylor \& Francis Group (Eds.), Handbook of Analysis of Active Compounds in Functional Foods (pp. 195-218).

Mazzeo, T., N'Dri, D., Chiavaro, E., Visconti, A., \& Fogliano, V. (2011) Effect of two cooking procedures on phytochemical compounds, total antioxidant capacity and colour of selected frozen vegetables. Food Chemistry, 128, 627-633.

Mukherjee, S., \& Chattopadhyay, P. K. (2007). Whirling bed blanching of potato cubes and its effects on product quality. Journal of Food Engineering, 78, 52-60.

Ohira, S. I., Kuhara, K., Shigetomi, A., Yamasaki, T., Kodama, Y., Dasgupta, P. K., \& Toda, K. (2014). On-line electrodialytic matrix isolation for chromatographic determination of organic acids in wine. Journal of Chromatography A, $1372,18-24$.

Rodriguez-Bernaldo de Quiros, A., Lage-Yusty, M.A., \& Lopez-Hernandez, J. (2009). HPLC analysis of organic acids using novel stationary phase. Talanta, 78, 643-646.

Scherer, R., Poloni Rybka, A. C., Ballus, C. A., Meinhart, A. D., Teixeira Filho, J., \& Teixeira Godoy, H. (2012) Validation of a HPLC method for simultaneous determination of main organic acids in fruits and juices. Food Chemistry, 135, 150-154.

Schnarreberger, C., \& Martin, W. (2002). Evolution of the enzymes of the citric acid cycle and the glyoxylate cycle of higher plants. European Journal of Biochemistry, 269, 868-883.

Soininen, T. H., Jukarainen, N., Auriola, S. O. K., Julkunen-Tiitto, R., Karjalainen, R., \& Vepsalainen, J. J. (2014). Quantitative metabolite profiling of edible onion species by NMR and HPLC-MS. Food Chemistry, 165, 499-505.

Theron, M. M., \& Rykers, J. F. (2010). Nature and composition of organic acids. In: CRC Press Taylor \& Francis Group (Eds.), Organic Acids and Food Preservation (pp. 21-50).

Turkmen, N., Sari, F., \& Velioglu, Y.S. (2005). The effect of cooking methods on total phenolics and antioxidant activity of selected green vegetables. Food Chemistry, 93, 713-718.

Wambui Munyaka, A., Oey, I., Van Loey, A., \& Hendrickx, M. (2010). Application of thermal inactivation of enzymes during vitamin $\mathrm{C}$ analysis to study the influence of acidification, crushing and blanching on vitamin $\mathrm{C}$ stability in Broccoli (Brassica oleracea L var. italica). Food Chemistry, 120, 591-598. 